On the validity of linear hydrodynamics for low-density granular flows described by the Boltzmann equation

This content has been downloaded from IOPscience. Please scroll down to see the full text. 1999 Europhys. Lett. 48359

(http://iopscience.iop.org/0295-5075/48/4/359)

View the table of contents for this issue, or go to the journal homepage for more

Download details:

IP Address: 150.214.182.194

This content was downloaded on 15/06/2015 at 18:21

Please note that terms and conditions apply. 
Europhys. Lett., 48 (4), pp. 359-364 (1999)

\title{
On the validity of linear hydrodynamics for low-density granular flows described by the Boltzmann equation
}

\author{
J. J. Brey, M. J. Ruiz-Montero and D. Cubero \\ Física Teórica, Universidad de Sevilla - Apartado de Correos 1065, E-41080 Sevilla, Spain
}

(received 29 March 1999; accepted in final form 15 September 1999)

PACS. 05.20-y - Classical statistical mechanics.

PACS. 05.20Dd - Kinetic theory.

PACS. 81.05Rm- Porous materials; granular materials.

\begin{abstract}
The direct Monte Carlo simulation method is used to check the validity of the hydrodynamic description of a low-density granular flow in the linear region about the homogeneous cooling state. Good agreement is found between the simulation results and the hydrodynamic equations obtained recently from the Boltzmann equation by using a generalized Chapman-Enskog procedure. In particular, the dependence of the transport coefficients on the coefficient of restitution predicted by the theory is verified.
\end{abstract}

Extended hydrodynamic equations, accounting for dissipation among the interacting particles, are frequently used to describe the macroscopic behavior of rapid granular flows [1]. As is the case for molecular fluids, the basis for such equations and explicit expressions for the coefficients appearing in them is provided by the kinetic theory for granular fluids. Nevertheless, even if the validity of the description provided by a given kinetic equation, e.g. the Boltzmann equation, is assumed, there is some controversy about the possibility of going from a kinetic theory to a hydrodynamic level of description by using a Chapman-Enskog expansion around the homogeneous cooling state beyond the quasielastic limit $[2,3]$. The cooling rate following from dissipation in collisions introduces a new time scale that is faster than the usual hydrodynamic scale. For instance, the thermal velocity associated to the temperature of the cooling reference state decays faster than the (inelastic) shear modes in the long-wavelength region. It could happen that for large inelasticity there were no time scale separation between the hydrodynamic and the purely kinetic contributions to the time evolution of the system, i.e. there were no "aging to hydrodynamics" or, in the kinetic theory language, "normal solution" of the kinetic equation [4]. Of course, a complete and definite answer to this question can only be obtained by solving exactly the corresponding kinetic equation, something that is far beyond the present perspectives. An interesting alternative is to construct numerical solutions to the Boltzmann equation and compare them with the corresponding solutions obtained by assuming the validity of a hydrodynamic description. The aim of this paper is to carry out such a comparison for states near the cooling reference state, i.e. in the so-called linear region. 
The system we will consider is a freely evolving low-density gas composed of smooth inelastic hard spheres of mass $m$, diameter $\sigma$, and a constant coefficient of normal restitution $\alpha$. The time evolution of the one-particle distribution function of the gas is assumed to be described by the (inelastic) Boltzmann equation [5,6], at least for the initial conditions and time intervals we will consider. Then, by using the Chapman-Enskog procedure, it is possible to obtain closed hydrodynamic evolution equations for the number density $n(\mathbf{r}, t)$, the flow velocity $\mathbf{u}(\mathbf{r}, t)$ and the local temperature $T(\mathbf{r}, t)[7]$

$$
\begin{gathered}
\partial_{t} n+\boldsymbol{\nabla} \cdot(n \mathbf{u})=0, \\
\partial_{t} \mathbf{u}+\mathbf{u} \cdot \boldsymbol{\nabla} \mathbf{u}+(m n)^{-1} \boldsymbol{\nabla} \cdot \mathrm{P}=0, \\
\partial_{t} T+\mathbf{u} \cdot \boldsymbol{\nabla} T+2\left(3 n k_{\mathrm{B}}\right)^{-1}(\mathrm{P}: \boldsymbol{\nabla} \mathbf{u}+\boldsymbol{\nabla} \cdot \mathbf{q})+T \zeta=0,
\end{gathered}
$$

where $k_{\mathrm{B}}$ is the Boltzmann constant, $\mathrm{P}(\mathbf{r}, t)=p \mathbf{I}-\eta\left[\boldsymbol{\nabla} \mathbf{u}+(\boldsymbol{\nabla} \mathbf{u})^{+}-\frac{2}{3} \mathbf{I} \boldsymbol{\nabla} \cdot \mathbf{u}\right]$ the pressure tensor, $\mathbf{q}(\mathbf{r}, t)=-\kappa \boldsymbol{\nabla} T-\mu \boldsymbol{\nabla} n$ the heat flux, and $\zeta(\mathbf{r}, t)$ the cooling rate due to energy dissipation in collisions. Here $p=n k_{\mathrm{B}} T$ is the hydrodynamic pressure, I the unit tensor, $\eta$ the shear viscosity, $\kappa$ the heat conductivity, and $\mu$ a new transport coefficient that has no analogue in the elastic case. The cooling rate has contributions of zeroth and second order in the gradients, $\zeta=\zeta^{(0)}+\zeta^{(2)}$. Of course, $\zeta^{(2)}$ has both linear and nonlinear in the gradients contributions, being the former ones of the form $\zeta_{1} \nabla^{2} T+\zeta_{2} \nabla^{2} n$. The coefficients $\eta, \kappa, \mu, \zeta^{(0)}, \zeta_{1}$, and $\zeta_{2}$ are functions of the coefficient of normal restitution $\alpha$. In the elastic limit, $\eta(\alpha)$ and $\kappa(\alpha)$ reduce to the Boltzmann values for elastic spheres $\eta_{0}$ and $\kappa_{0}$, while all the other coefficients vanish. The explicit expressions can be found in ref. [7] and will not be given here. Equivalent hydrodynamic equations but restricted to the low dissipation limit have been derived recently by Sela and Goldhirsch [8].

The above hydrodynamic equations have an exact solution describing the so-called homogeneous cooling state (HCS), given by $\mathbf{u}_{\mathrm{H}}=0, \boldsymbol{\nabla} n_{\mathrm{H}}=\boldsymbol{\nabla} T_{\mathrm{H}}=0$, and $T_{\mathrm{H}}(t)=$ $T_{\mathrm{H}}(0)\left[1+\zeta^{(0)}(0) t / 2\right]^{-2}$. To study the time evolution of small deviations from the HCS, the hydrodynamic equations are linearized about the fields defining the state. This leads to partial differential equations with coefficients that do not depend on space but are time dependent. Thus it is convenient to introduce new time and space variables by $\mathrm{d} \tau=\nu_{\mathrm{H}}(t) \mathrm{d} t / 2$ and $\mathrm{d} \mathbf{l}=\nu_{\mathrm{H}}(t) v_{\mathrm{H}}^{-1}(t) \mathrm{d} \mathbf{r} / 2$, where $\nu_{\mathrm{H}}(t)=16 n_{\mathrm{H}} \sigma^{2} \pi^{1 / 2} v_{\mathrm{H}}(t) / 5$ and $v_{\mathrm{H}}=\left[k_{\mathrm{B}} T_{\mathrm{H}}(t) / m\right]^{1 / 2}$. Note that $\nu_{\mathrm{H}}$ is proportional to the Boltzmann collision frequency at the temperature $T_{\mathrm{H}}(t)$, that is close but not exactly the same as the collision frequency in the HCS, due to the distortion of the velocity distribution with respect to a Gaussian. Also we define scaled hydrodynamic fields as $\rho(\mathbf{l}, \tau)=\delta n(\mathbf{l}, \tau) / n_{\mathrm{H}}, \boldsymbol{\omega}(\mathbf{l}, \tau)=\delta \mathbf{u}(\mathbf{l}, \tau) / v_{\mathrm{H}}(\tau)$, and $\theta(\mathbf{l}, \tau)=\delta T(\mathbf{l}, \tau) / T_{\mathrm{H}}(\tau)$. Here $\delta y(\mathbf{l}, \tau)$ denotes the local deviation of the field $y$ from its value in the HCS, $y_{\mathrm{H}}$. Next we transform to Fourier space $\mathbf{k}$ with respect to the scaled space variable $\mathbf{l}$. It is found that the transversal components of the velocity field relative to the wave vector, $\boldsymbol{\omega}_{\mathbf{k} \perp}$, are decoupled from the other fields and behave as

$$
\boldsymbol{\omega}_{\mathbf{k} \perp}(\tau)=\boldsymbol{\omega}_{\mathbf{k} \perp}(0) \exp \left[s_{\perp} \tau\right], \quad s_{\perp}=\zeta^{*}-\eta^{*} k^{2} / 2,
$$

where $\zeta^{*}=\zeta_{\mathrm{H}}^{(0)} / \nu_{\mathrm{H}}$ and $\eta^{*}=\eta / \eta_{0}$ are functions of $\alpha$ alone. The Fourier components of the remaining hydrodynamic fields obey the set of coupled equations [7]

$$
\begin{gathered}
\partial_{\tau} \rho_{\mathbf{k}}+i k \omega_{\mathbf{k} \|}=0 \\
\left(\partial_{\tau}-\zeta^{*}+2 \eta^{*} k^{2} / 3\right) \omega_{\mathbf{k} \|}+i k \theta_{k}+i k \rho_{k}=0 \\
{\left[\partial_{\tau}+\zeta^{*}+5\left(\kappa^{*}-\zeta_{1}^{*}\right) k^{2} / 4\right] \theta_{k}+\left[2 \zeta^{*}+5\left(\mu^{*}-\zeta_{2}^{*}\right) k^{2} / 4\right] \rho_{\mathbf{k}}+2 i k \omega_{\mathbf{k} \|} / 3=0 .}
\end{gathered}
$$


Here $\omega_{\mathbf{k} \|}$ is the longitudinal component of $\boldsymbol{\omega}_{\mathbf{k}}$ and we have introduced the reduced coefficients $\kappa^{*}=\kappa / \kappa_{0}, \zeta_{1}^{*}=3 p_{\mathrm{H}} \zeta_{1} / 2 \kappa_{0}$ and $\zeta_{2}^{*}=3 n_{\mathrm{H}}^{2} k_{\mathrm{B}} \zeta_{2} / 2 \kappa_{0}$. Again these coefficients depend only on the coefficient of normal restitution $\alpha$. The linear stability analysis of eqs. (5)-(7) predicts that, if $k<k_{\|}=4 \zeta^{*} / 5\left(\kappa^{*}-\zeta_{1}^{*}-\mu^{*}+\zeta_{2}\right)$, after an initial transient the time dependence of the Fourier transform of the scaled hydrodynamics fields $\rho_{\mathbf{k}}, \theta_{\mathbf{k}}$ and $\omega_{\mathbf{k} \|}$, will be of the form $\sim \exp \left[s_{\|} \tau\right]$, where $s_{\|}$is the real (and positive) eigenvalue of the eigensystem defined by eqs. (5)-(7). The quantity $s_{\|}$depends on $k$ and also on the coefficient of restitution $\alpha$ through the reduced coefficients defined above [7].

In order to test the accuracy of the above linear hydrodynamic equations to describe the time evolution of a gas obeying the inelastic Boltzmann equation near the HCS, we have obtained numerical solutions by using the direct Monte Carlo simulation method [9]. We have considered a system of size $L$ in the direction in which the gradient of the hydrodynamics fields will be studied, taken as $X$-axis. In this direction, periodic boundary conditions have been applied, while the system was considered as homogeneous with respect to the $y$ and $z$ coordinates. Initially, the particles were distributed uniformly and with a Gaussian velocity distribution. After a short transient time, the system reached the HCS [10]. Then, an external perturbation was introduced at a given time taken as $t=0$. The simulations typically involved $N=2 \cdot 10^{5}$ particles and the data were averaged over 100 trajectories. Of course, in the simulation there is no intermediate step that relies on the existence of hydrodynamic equations.

First, let us describe the results after an initial perturbation of the transversal component of the velocity,

$$
u_{y}(x, 0)=u_{0} \sin \left(q_{0} x\right)
$$

where $q_{0}=2 \pi / L$. The study of a perturbation of the transversal velocity field is of particular importance because, although it is decoupled in the linear approximation of the other hydrodynamic fields, it has been suggested [11] that its nonlinear coupling to them is responsible for the clustering occurring in granular media. Figure 1 shows the profile $u_{y}(x)$ at three different times in a typical simulation. The values of the parameters in this case were $\alpha=0.85$ and $L=50 \lambda$, with $\lambda=1 / \sqrt{2} n \pi \sigma^{2}$ being the mean free path. The amplitude of the initial perturbation was $u_{0}=0.1\left[2 k_{\mathrm{B}} T_{\mathrm{H}}(0) / m\right]^{1 / 2}$. The profile is accurately fitted by a sine function $u_{y}(x, \tau)=u_{\tau} \sin \left(q_{0} x\right)$ in all the time interval considered. This is characteristic of the linear regime as described by the above hydrodynamic equations, that predicts $u_{\tau}=u_{0} \exp \left[-\eta^{*} k_{0}^{2} \tau / 2\right], k_{0}=2 \nu_{\mathrm{H}}^{-1}(t) v_{\mathrm{H}}(t) q_{0}$. For the simulation considered in fig. 1, $k_{0}=0.1969$, and the solid lines have been plotted by using the theoretical prediction. We emphasize that the bare velocity $\mathbf{u}(\tau)$ always decays in time, while the scaled one can increase for large enough dissipation. In particular, for the parameters used in the simulation reported in fig. 1, eq. (4) predicts that $\omega_{y}$ grows in time. This is related with the formation of density clusters in freely evolving granular fluids [11].

To get a more quantitative comparison between the simulation results and the theory, we have fitted numerically the transversal velocity profile to a sine curve of wavelength $L$ at several times. The amplitude of such sine function decayed exponentially with $\tau$, and the fit to an exponential of this amplitude provides the numerical value of the reduced shear viscosity $\eta^{*}(\alpha)$. We have repeated the simulations for several values of $\alpha$ in the interval $0.625 \leq \alpha \leq 0.925$. For smaller values the analysis is very hard, since the system becomes unstable very soon and not reliable statistics can be obtained, but in all the cases there is a relevant time window where linear hydrodynamics predicts accurately the results of the simulations. The numerical values for the shear viscosity are compared with the theoretical predictions of ref. [7] in fig. 2. A first conclusion emerging from the results is that there is a relevant dependence of the reduced shear viscosity on the coefficient of restitution, namely the former increases monotonically when the 


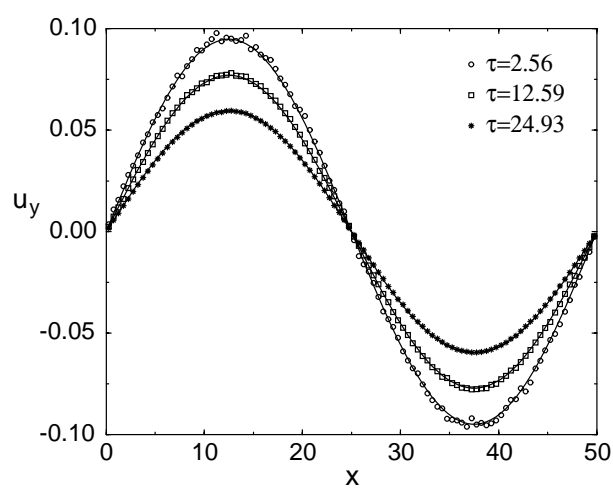

Fig. 1

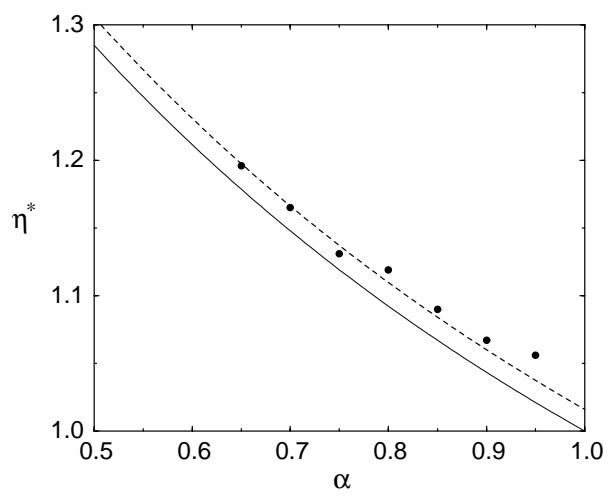

Fig. 2

Fig. 1. - Transversal velocity profile at three different times after an initial harmonic perturbation. The velocity is measured in units of $\left(2 k_{\mathrm{B}} T(0) / m\right)^{1 / 2}$ and $x$ in units of the mean free path $\lambda$. The symbols are from the simulation and the solid lines the theoretical predictions.

Fig. 2. - Reduced shear viscosity $\eta^{*}$ as a function of the coefficient of normal restitution $\alpha$. The points are from the direct Monte Carlo simulation and the solid line the theoretical expression derived in ref. [7]. The dashed line is obtained by multiplying the theoretical value by the constant factor 1.016 obtained in the elastic limit.

latter decreases. For $\alpha=0.8$, this reduction is already of the order of $10 \%$. Moreover, although the theory predictions agree fairly well with the simulation values, there is a small systematic deviation. We believe that it is due in part to the approximations introduced in applying the Chapman-Enskog procedure, and more concretely to the use of the first Enskog approximation. In fact, it is known that a next-order calculation in the Sonine expansion gives a correction factor of the order of 1.016 for the shear viscosity in the elastic limit $\alpha=1$ [12]. If this factor were taken into account, a better agreement between theory and simulation would be obtained (see dashed line in fig. 2). Another source of discrepancy is the growing amplitude of the scaled perturbation $\omega_{\perp}$, which ultimately invalidates the linear analysis.

Of course, it could be argued that the transversal velocity is a very special hydrodynamic field and that a more general perturbation should be presented to test the validity of the linear hydrodynamic description. For this reason we have analyzed the time evolution of the hydrodynamic fields after an initial perturbation of the form

$$
\rho(x, 0)=\rho_{0} \sin \left(q_{0} x\right),
$$

where $q_{0}$ is the same as above. This perturbation has components along the three eigenvectors of the matrix associated to the linear problem defined by eqs. (5)-(7). Then, not only the density but also the temperature and the longitudinal component of the velocity deviate from the reference values for $t>0$. In the linear regime they are given by harmonic functions with wave number $q_{0}$. In fig. 3 the time evolution of the Fourier amplitudes of the involved hydrodynamic fields is shown for a perturbation given by eq. (9) with $\rho_{0}=0.1, \alpha=0.8$ and $L=50 \lambda$. The other parameters of the simulations are the same as in fig. 1. The solid lines have been obtained by solving numerically the linearized hydrodynamic equations. The agreement with the simulation results from the Boltzmann equation is excellent and extends over a quite large time interval. Note that the time scale $\tau$ is of the order of the accumulate number of collisions per particle. Since the solutions represented in the figure involved the three coupled 


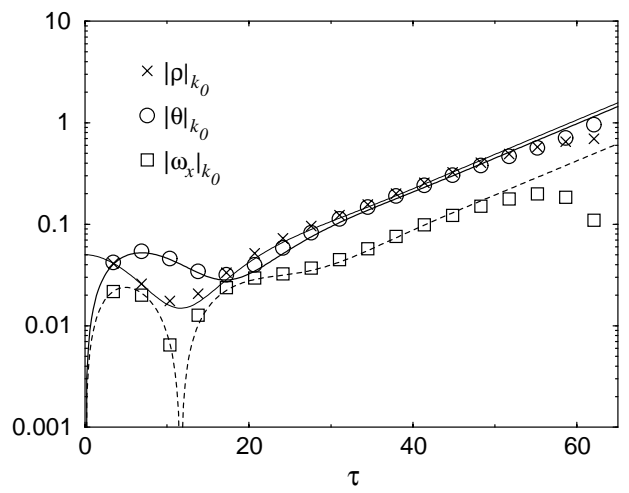

Fig. 3

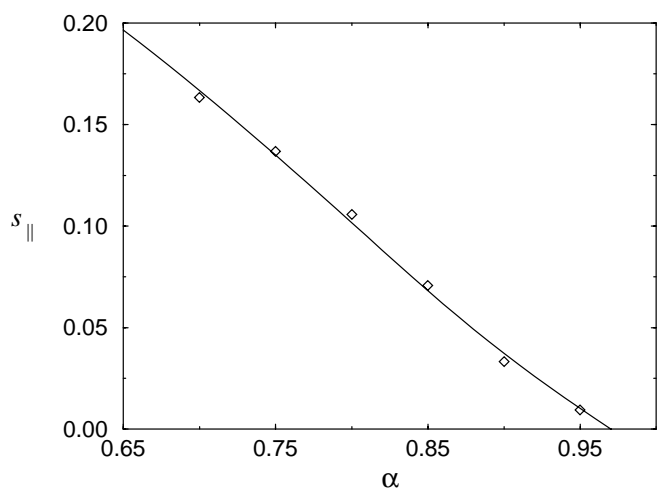

Fig. 4

Fig. 3. - Time evolution of the Fourier components of the scaled dimensionless density $\rho$, temperature $\theta$, and longitudinal velocity $\omega_{x}$, following a small harmonic perturbation of the density. The symbols are results from the simulations and the lines the predictions from linear hydrodynamics.

Fig. 4. - The eigenvalue $s_{\|}$for different values of the restitution coefficient and $k=0.1406$, equivalent to $L=70 \lambda$. The symbols are the results of the Monte Carlo simulation and the continuous line the theoretical prediction from the hydrodynamic equations.

equations (5)-(7) and the shape of the curves is far from being trivial, the results strongly support the validity of hydrodynamics to describe the time evolution of a low-density granular fluid, at least in the vicinity of the HCS.

From fig. 3 it also follows that the three plotted hydrodynamic fields grow exponentially after an initial transient, and that the coefficient characterizing the exponential growth is the same for the three of them. This is consistent with hydrodynamics, and the fit to an exponential of this long time behavior provides the simulation value for $s_{\|}$. We have repeated this study for different values of $\alpha$ in the interval $0.7 \leq \alpha \leq 0.95$ keeping the initial perturbation the same as in eq. (9). In all the cases the behavior of the system was well described by the hydrodynamic equations, and became exponential after an initial transient. In fig. 4 we have plotted $s_{\|}$obtained from the simulations as a function of $\alpha$ for fixed $k$, and compared it with the theoretical prediction of eqs. (5)-(7). The agreement is quite remarkable, and shows that the capability of hydrodynamics to describe qualitatively and quantitatively the behavior of rapid low-density granular flows extends over a wide range of values of the restitution coefficient. It must be noticed that $s_{\|}$depends on all the reduced transport and source coefficients. Therefore, the good agreement shown in fig. 4 implies also the accuracy of the expressions for them derived in ref. [7], a conclusion that is clearly not restricted to the limit of small inelasticity. Deltour and Barrat [13] studied as well $s_{\|}$as a function of $k$ keeping $\alpha$ fixed through the structure factor for a system of hard disks by Molecular Dynamic simulation, concluding that hydrodynamics predicts correctly the observations of their simulations. In any case, the determination of the explicit range of validity of linear hydrodynamics as a function of wave number, frequency and dissipation would require very extensive simulations. We believe that this work shows that linear hydrodynamics holds to describe the initial evolution of small perturbations even for rather inelastic systems.

In this letter we have restricted ourselves to the linear region about the HCS. As a consequence, we have not considered the development of density instabilities in the system and nonlinear couplings between hydrodynamic modes. In this context, let us note that, although 
$s_{\|}$is positive, the density is not observed to grow monotonically at short times in fig. 3, due to the contributions from the other two modes. These points are discussed in detail elsewhere [14]. A related question is the influence of periodic boundary conditions and the consideration of initial perturbations with the maximum allowed wavelength. In principle, this comment applies both to elastic and to inelastic systems. Nevertheless, inelasticity has as a consequence that the dominant modes are those corresponding to the largest wavelength (shortest wave number) $[7,8]$. Therefore, the perturbations we have introduced are the most relevant for the evolution of the system in the linear region. In fact, these are the dominant spontaneous perturbations in the system [14].

$* * *$

It is a pleasure to thank J. W. DufTy for helpful discussions. Partial support by Grant

No. PB98-1124 from the Dirección General de Investigación Científica y Técnica (Spain) is acknowledged.

\section{REFERENCES}

[1] Haff P. K., J. Fluid Mech., 134 (1983) 401; Campbell C. S., Annu. Rev. Fluid Mech., 22 (1990) 57.

[2] Kadanoff L. P., Rev. Mod. Phys., 71 (1999) 435.

[3] Tan M. L. and Goldhirsch I., Phys. Rev. Lett., 81 (1998) 3022.

[4] McLennan J. A., Introduction to Nonequilibrium Statistical Mechanics (Prentice-Hall, New Jersey) 1989.

[5] Goldshtein A. and Shapiro M., J. Fluid Mech., 282 (1995) 75.

[6] Brey J. J., Dufty J. W. and Santos A., J. Stat. Phys., 87 (1997) 1051.

[7] Brey J. J., Dufty J. W., Kim C. S. and Santos A., Phys. Rev. E, 58 (1998) 4638.

[8] Sela N. and Goldhirsch I., J. Fluid Mech., 361 (1998) 41.

[9] Bird G., Molecular Gas Dynamics and the Direct Simulation of Gas Flows (Clarendon Press, Oxford) 1994.

[10] Brey J. J., Ruiz-Montero M. J. and Cubero D., Phys. Rev. E, 54 (1996) 3664.

[11] Goldhirsch I., Tan M. L. and Zanetti G., Phys. Rev. Lett., 70 (1993) 1619; McNamara S. and Young W. R., Phys. Fluids, 5 (1993) 34; Phys. Rev. E, 53 (1996) 5089.

[12] Gass D. M., J. Chem. Phys., 54 (1971) 1898.

[13] Deltour P. and Barrat J. L., J. Phys. I, 7 (1997) 137.

[14] Brey J. J., Ruiz-Montero M. J. and Cubero D., Phys. Rev. E, 60 (1999) 3150. 